\title{
Association between human $T$ cell leukemia virus type-1 (HTLV-1) infection and advanced periodontitis in relation to atherosclerosis among elderly Japanese: a cross-sectional study
}

Yuji Shimizu ${ }^{1,2^{*}}$ D, Hirotomo Yamanashi ${ }^{3}$, Masayasu Kitamura ${ }^{4}$, Reiko Furugen ${ }^{4}$, Takahiro Iwasaki ${ }^{5}$, Hideki Fukuda ${ }^{4}$, Hideaki Hayashida ${ }^{4}$, Koji Kawasaki ${ }^{6}$, Kairi Kiyoura', Shin-Ya Kawashiri ${ }^{1}$, Toshiyuki Saito ${ }^{4}$, Atsushi Kawakami ${ }^{7}$ and Takahiro Maeda ${ }^{1,3,8}$

\begin{abstract}
Background: Human T cell leukemia virus type-1 (HTLV-1) stimulates inflammation activity. Our previous study revealed a positive association between asymptomatic HTLV-1 infection and advanced periodontitis among elderly Japanese individuals with low levels of hematopoietic activity (reflected by reticulocyte levels). Since low hematopoietic activity has been correlated with low-grade inflammation and low-grade inflammation is associated with atherosclerosis, the status of atherosclerosis could, in turn, determine the nature of this association.

Methods: To this end, a cross-sectional study of 907 elderly Japanese individuals (aged 60-99 years), who had participated in dental health check-up during the period 2016-2018, was conducted. Advanced periodontitis was defined as periodontal pocket $\geq 6.0 \mathrm{~mm}$.
\end{abstract}

Results: Among the study population, 295 (32.5\%) were found to have atherosclerosis defined as a carotid intimamedia thickness (CIMT) of $\geq 1.1 \mathrm{~mm}$. HTLV-1 infection was positively associated with advanced periodontitis in participants with atherosclerosis, but no significant associations were observed among the participants without atherosclerosis. The known risk factors' (including reticulocyte and CIMT) adjusted odds ratio (OR) and 95\% confidence interval (Cl) of advanced periodontitis were OR 2.01 and 95\% Cl 1.06-3.81 for participants with atherosclerosis and $\mathrm{OR} 0.61$ and $95 \% \mathrm{Cl} 0.34-1.12$ for participants without atherosclerosis.

Conclusion: This study found a significant association between HTLV-1 infection and advanced periodontitis among elderly Japanese with atherosclerosis. However, this association is absent in individuals without atherosclerosis, suggesting that atherosclerosis might act as a determinant in the association between HTLV-1 infection and advanced periodontitis among elderly Japanese.

Keywords: Atherosclerosis, Elderly, HTLV-1, Periodontitis

\footnotetext{
* Correspondence: shimizu@osaka-ganjun.jp

${ }^{1}$ Department of Community Medicine, Nagasaki University Graduate School

of Biomedical Sciences, Nagasaki-shi, Sakamoto 1-12-4, Nagasaki 852-8523,

Japan

${ }^{2}$ Department of Cardiovascular Disease Prevention, Osaka Center for Cancer

and Cardiovascular Disease Prevention, Osaka, Japan

Full list of author information is available at the end of the article
}

(c) The Author(s). 2019 Open Access This article is distributed under the terms of the Creative Commons Attribution 4.0 International License (http://creativecommons.org/licenses/by/4.0/), which permits unrestricted use, distribution, and reproduction in any medium, provided you give appropriate credit to the original author(s) and the source, provide a link to the Creative Commons license, and indicate if changes were made. The Creative Commons Public Domain Dedication waiver (http://creativecommons.org/publicdomain/zero/1.0/) applies to the data made available in this article, unless otherwise stated. 


\section{Background}

Although human $\mathrm{T}$ cell leukemia virus type-1 (HTLV-1), which is the earliest recognized human retrovirus, has been shown to be a causative agent of adult $\mathrm{T}$ cell leukemia/lymphoma [1], as well as associated with myelopathy/tropical spastic paraparesis, sensorimotor polyneuropathy, and optic neuritis [2], the majority of carriers remain asymptomatic throughout their lives [3-5].

However, we have previously reported the positive association of asymptomatic HTLV-1 infection to advanced periodontitis in elderly subjects with low hematopoietic activity (and low reticulocyte levels) [6]. Although HTLV-1 enhances inflammation activity [7, 8], the factor that activates HTLV-1 is unknown. However, the influence of HTLV-1 infection should be stronger in participants with inflammation than in those without inflammation. Severe cases of chronic periodontitis were associated with endothelial (microvascular) dysfunction $[9,10]$. Therefore, the presence of inflammation associated with endothelial dysfunction might act as a determinant factor in the association between HTLV-1 infection and advanced periodontitis. As low hematopoietic activity is associated with low-grade inflammation [11-14], elderly subjects with low hematopoietic activity could assume an important role in the association between HTLV-1 infection and advanced periodontitis [6].

In addition, low-grade inflammation is associated with atherosclerosis [15] and subsequent disruption of microcirculation [16]. Therefore, asymptomatic HTLV-1 infection could be positively associated with advanced periodontitis in subjects diagnosed with developing atherosclerosis.

Since periodontitis is a major cause of tooth loss which results in a significant decrease in the quality of life of elderly people [17] and HTLV-1 is known to be a common retrovirus [18], clarifying the association between HTLV-1 infection and advanced periodontitis in relation to atherosclerosis is important in preserving the quality of life among elderly population. However, we are unaware of any study that has clarified the associations between HTLV-1 infection and advanced periodontitis in relation to the status of atherosclerosis.

To evaluate the association between HTLV-1 infection and advanced periodontitis in relation to atherosclerosis status, we conducted a cross-sectional study involving 907 elderly people aged 60-99 years who participated in annual dental health check-ups during 2016-2018.

\section{Methods}

\section{Study population}

The study population comprised of 1925 elderly Japanese (702 men and 1223 women) aged 60-99 years from
Goto City in the western part of Japan who attended annual health check-ups during 2016-2018 conducted by the local government and directed by the Ministry of Health, Labor and Welfare in Japan and who underwent oral assessment.

Participants without data on carotid intima-media thickness (CIMT) $(n=4)$ and those without any remaining teeth $(n=920)$ were excluded. Advanced periodontitis is diagnosed as the maximum probing pocket depth for each gingiva. Incidentally, decayed teeth are the most frequent reason for teeth extraction and subsequent periodontal diseases in Japan [19]. Therefore, a small number of participants with normal teeth might induce a detection bias for periodontitis. On this basis, subjects with $1-4$ remaining teeth $(n=90)$, as well as people with missing HTLV-1 data $(n=3)$ and missing reticulocyte data $(n=1)$, were excluded. The remaining participants comprised of 353 men and 554 women with a mean age of 72.1 (standard deviation [SD] 7.0) for men and 71.3 (SD 6.8) for women were enrolled in the study.

\section{Data collection and laboratory measurements}

Trained interviewers obtained information on smoking status. Body weight and height in bare feet and light closing were measured by using an automatic body composition analyzer (BF-220; Tanita, Tokyo, Japan). Body mass index (BMI) was calculated as weight $(\mathrm{kg}) /$ height $(\mathrm{m})^{2}$.

Blood samples were collected with siliconized, sodium fluoride, and EDTA-2K containing tubes. All measurements were made following standard laboratory procedures at SRL, Inc. (Tokyo, Japan). Samples from the siliconized tube were used to measure high-density lipoprotein (HDL)-cholesterol and triglycerides, and sample from the sodium fluoride tube was used to measure glycated hemoglobin (HbA1c). Red blood cells (RBC) and reticulocytes in the samples collected in EDTA-2K tubes were measured. Reticulocyte levels were determined using the following formula: reticulocytes $\left(\times 10^{4}\right.$ cells/ $\mu \mathrm{L})=($ reticulocytes, $\%) \times \mathrm{RBC} \quad\left(\times 10^{4} \quad\right.$ cells $\left./ \mu \mathrm{L}\right) / 1000$. The calculated values were $5.358 \pm 2.141\left[\times 10^{4}\right.$ cells $\left./ \mu \mathrm{L}\right]$ and $5.047 \pm 1.774\left[\times 10^{4}\right.$ cells $\left./ \mu \mathrm{L}\right]$ for male and female participants, respectively.

\section{Measurement of carotid intima media-thickness}

Experienced vascular technicians measured carotid intima media-thickness (CIMT) using a LOGIQ Book XP with a $10-\mathrm{MHz}$ transducer (GE Healthcare, Milwaukee, WI, USA). Maximum values for the left and right common carotid arteries of the CIMT were calculated with automated digital edge-detection software (Intimascope; MediaCross, Tokyo, Japan) following a protocol that has been described in detail elsewhere [20]. The higher values of right and left CIMT not including plaque 
measurements were then calculated, and the maximum CIMT value was used for analysis. Since a previous study reported a CIMT value of $<1.1 \mathrm{~mm}$ as normal [21], we defined samples with a CIMT value of $\geq 1.1 \mathrm{~mm}$ as atherosclerotic.

\section{Measurement of human T cell leukemia virus type- 1 (HTLV-1)}

To detect human $\mathrm{T}$ cell leukemia virus type-1 (HTLV1), a chemiluminescent enzyme immunoassay (CLEIA) kit (Fujirebio Inc., Tokyo, Japan) was used.

\section{Oral examination}

Trained dentists $(n=6)$ performed periodontal examination by using a modified method from the Third National Health and Nutrition Examination Survey (USA) [22]. Probing pocket depth was measured using a periodontal probe at the mesiobuccal and mild-buccal sites for all present teeth excluding the third molars. Prior to the start of this study, all examiners were trained and calibrated using a chart, periodontal models, and volunteers at the Nagasaki University Hospital.

\section{Statistical analysis}

Atherosclerosis was diagnosed as having CIMT $\geq 1.1$ $\mathrm{mm}$, and the study population was stratified into those with and without atherosclerosis. Atherosclerosis statusspecific differences in mean values or proportions of variables by HTLV-1 status were analyzed. A trend test was performed with analysis of variance (ANOVA) for continuous values, and chi-squared test was used for determining differences in proportions. Advanced periodontitis was diagnosed as a probing pocket depth of $\geq$ $6 \mathrm{~mm}$.

Logistic regression models were used to calculate odds ratios (ORs) and 95\% confidence interval (CIs) to determine the association between HTLV-1 infection and advanced periodontitis. Since atherosclerosis might act as a determinant factor on the association between HTLV-1 infection and advanced periodontitis as we hypothesized in the present study, participants were stratified according to their atherosclerosis status.

Periodontitis risk factors that have a direct influence on the intra-oral environment, such as smoking [23], and the number of remaining teeth were regarded as confounding factors. While no significant correlation between caries (decayed teeth) and periodontitis was observed in previous studies, both conditions have a common etiological factor [24, 25]. Further, decayed teeth are the most frequent reason for teeth extraction and subsequent periodontal diseases in Japan [19]. Hence, we also included decayed teeth as a confounding factor in our analysis. CIMT and reticulocytes were included in the analysis as confounding factors due to their possible mechanistic role in the development of atherosclerosis. Obesity defined by BMI and diabetes are known risk factors for periodontitis [26, 27]. Moreover, while no association between dyslipidemia and periodontitis has been reported [27], hypo-HDL cholesterol and hyper-triglycerides could be associated with periodontitis [28]. Thus, we included BMI, HbA1c, HDL cholesterol, and triglycerides as confounding factors in our logistic regression models. Further, since medication for diabetes [29] and hyperlipidemia [30] might influence the severity of periodontitis, we included these as confounding factors.

Three different models were used to adjust for confounding factors. Model 1 was only adjusted for sex and age. For model 2, we included other potential confounding factors, namely BMI $\left(\mathrm{kg} / \mathrm{m}^{2}\right)$, smoking status (nonsmoker or current smoker), number of remaining teeth, decayed teeth (presence, absence), HDL cholesterol (mg/ $\mathrm{dL})$, triglycerides $(\mathrm{mg} / \mathrm{dL})$, HbA1c (\%), reticulocyte $($ cells $/ \mu \mathrm{L})$, and CIMT $(\mathrm{mm})$. Model 3 was further adjusted for the use of glucose-lowering medication (yes, no) and antihyperlipidemic medication (yes, no).

In addition, we evaluated the influence of atherosclerosis on the association between HTLV-1 infection and advanced periodontitis by logistic regression analysis.

Furthermore, we also performed sex-specific analyses to determine the association between HTLV-1 infection and advanced periodontitis according to the status of atherosclerosis.

As reticulocyte levels have an ambivalent association with hypertension (systolic blood pressure $\geq 140 \mathrm{mmHg}$ and/or diastolic blood pressure $\geq 90 \mathrm{mmHg}$ and/or having used antihypertensive medication) and atherosclerosis in the elderly [31], we also conducted analyses of the relationship between reticulocytes (tertile values) and hypertension and that of reticulocytes (tertile values) and atherosclerosis using a sex- and age-adjusted model.

All statistical analyses were performed with SAS system for Windows (version 9.4: SAS Inc., Cary, NC). A $p$ value of $<0.05$ was considered statistically significant.

\section{Results}

Of the study population, 295 and 197 were diagnosed as having atherosclerosis and advanced periodontitis, respectively.

\section{Characteristics of the study population}

Among the participants without atherosclerosis, people who were HTLV-1 positive were older compared to those who were HTLV-1 negative. Among the participants with atherosclerosis, HTLV-1 positive individuals had significantly lower triglycerides and HbA1c levels than people who were HTLV-1 negative (Table 1). 
Table 1 Characteristics of the study population

\begin{tabular}{|c|c|c|c|c|c|c|}
\hline & \multicolumn{6}{|c|}{ Atherosclerosis } \\
\hline & \multicolumn{3}{|l|}{$(-)$} & \multicolumn{3}{|l|}{$(+)$} \\
\hline & \multicolumn{2}{|c|}{ HTLV-1 infection } & \multirow[t]{2}{*}{$p$} & \multicolumn{2}{|c|}{ HTLV-1 infection } & \multirow[t]{2}{*}{$p$} \\
\hline & $\overline{(-)}$ & $(+)$ & & $(-)$ & $(+)$ & \\
\hline No. of subjects & 512 & 100 & & 232 & 63 & \\
\hline Men, \% & 36.3 & 31.0 & 0.309 & 47.8 & 39.7 & 0.251 \\
\hline Age, year & $70.1 \pm 6.5$ & $72.0 \pm 6.6$ & 0.008 & $73.7 \pm 6.8$ & $75.6 \pm 6.9$ & 0.055 \\
\hline Body mass index (BMI), $\mathrm{kg} / \mathrm{m}^{2}$ & $22.7 \pm 3.2$ & $23.4 \pm 3.4$ & 0.043 & $23.4 \pm 3.4$ & $22.8 \pm 3.1$ & 0.151 \\
\hline Serum HDL cholesterol, mg/dL & $63 \pm 15$ & $60 \pm 16$ & 0.062 & $61.3 \pm 13.7$ & $63.8 \pm 15.6$ & 0.223 \\
\hline Serum triglycerides, mg/dL & $103 \pm 52$ & $111 \pm 61$ & 0.188 & $99 \pm 46$ & $84 \pm 30$ & 0.013 \\
\hline Hemoglobin A1c (HbA1c), \% & $5.7 \pm 0.5$ & $5.8 \pm 0.5$ & 0.060 & $5.9 \pm 0.6$ & $5.7 \pm 0.4$ & 0.007 \\
\hline Glucose lowering medication use, \% & 3.9 & 6.0 & 0.343 & 10.8 & 31.1 & 0.064 \\
\hline Antihyperlipidemic medication use, \% & 19.5 & 20.0 & 0.914 & 25.9 & 20.6 & 0.396 \\
\hline Current smoker, \% & 6.3 & 6.0 & 0.925 & 6.0 & 6.3 & 0.927 \\
\hline No. of remaining teeth & $22.0 \pm 6.4$ & $22.0 \pm 6.0$ & 0.999 & $20.3 \pm 6.6$ & $20.2 \pm 7.4$ & 0.923 \\
\hline Individuals with decayed teeth, \% & 29.1 & 31.0 & 0.704 & 30.6 & 25.4 & 0.423 \\
\hline CIMT, mm & $0.83 \pm 0.10$ & $0.84 \pm 0.10$ & 0.405 & $1.17 \pm 0.18$ & $1.15 \pm 0.22$ & 0.553 \\
\hline Reticulocyte, $\times 10^{4}$ cells $/ \mu \mathrm{L}$ & $5.169 \pm 1.806$ & $5.280 \pm 1.740$ & 0.574 & $5.071 \pm 1.663$ & $5.331 \pm 3.510$ & 0.405 \\
\hline
\end{tabular}

Values: mean \pm 1 standard deviation (SD). CIMT Carotid Intima-Media Thickness. Atherosclerosis is defined as carotid intima-media thickness (CIMT) $\geq 1.1$ mm.

Participants with advanced periodontitis show significantly higher values of BMI and decayed teeth (Table 2).

\section{Association between advanced periodontitis and HTLV-1 infection among total participants}

From the logistic regression models, the ORs and 95\% CIs for the correlation of advanced periodontitis to HTLV-1 infections among all participants are shown in Table 3. No significant association between HTLV-1 infection and advanced periodontitis were observed.

\section{Association between advanced periodontitis and HTLV-1 infection stratified by atherosclerosis status}

Atherosclerosis-status-specific ORs and 95\% CIs for advanced periodontitis in relation to HTLV-1 infections are presented in Table 4 . No significant association between HTLV-1 and advanced periodontitis was observed for participants without atherosclerosis. However, in the participants with atherosclerosis, being positive for HTLV-1 infection was associated with a greater likelihood of advanced periodontitis. An investigation into the effects of the associations between HTLV-1 infection and atherosclerosis on advanced periodontitis revealed significant interactions.

Sex-specific association between advanced periodontitis and HTLV-1 infection stratified by atherosclerosis status Sex-specific ORs and 95\% CIs for advanced periodontitis regarding HTLV-1 infection according to the status of atherosclerosis are shown in Table 5. Even the statistical
Table $\mathbf{2}$ Characteristics of the study population

\begin{tabular}{llll}
\hline & \multicolumn{3}{l}{ Advanced periodontitis } \\
\cline { 2 - 4 } & $(-)$ & $(+)$ & $p$ \\
\hline No. of subjects & 710 & 197 & \\
Men, \% & 36.6 & 47.2 & 0.007 \\
Age, year & $71.5 \pm 7.0$ & $72.3 \pm 6.3$ & 0.147 \\
HTLV-1 infection, \% & 17.7 & 18.8 & 0.738 \\
Body mass index (BMI), kg/m ${ }^{2}$ & $22.8 \pm 3.2$ & $23.6 \pm 3.4$ & 0.002 \\
Serum HDL cholesterol, mg/dL & $62 \pm 15$ & $61 \pm 15$ & 0.454 \\
Serum triglycerides, mg/dL & $101 \pm 50$ & $101 \pm 53$ & 0.908 \\
Hemoglobin A1c (HbA1c), \% & $5.8 \pm 0.5$ & $5.8 \pm 0.5$ & 0.953 \\
Glucose lowering & 5.5 & 7.1 & 0.394 \\
medication use, \% & & & \\
Antihyperlipidemic & 21.3 & 21.3 & 0.987 \\
medication use, \% & & & \\
Current smoker, \% & 5.4 & 9.1 & 0.051 \\
No. of remaining teeth & $21.6 \pm 6.6$ & $20.8 \pm 6.3$ & 0.123 \\
Individuals with decayed & 27.0 & 38.1 & 0.003 \\
teeth, \% & & & \\
ClMT, mm & $0.94 \pm 0.21$ & $0.96 \pm 0.20$ & 0.200 \\
Reticulocyte, $\times 10^{4}$ cells/ $\mu \mathrm{L}$ & $5.152 \pm 1.771$ & $5.223 \pm 2.422$ & 0.650 \\
\hline Valves men \pm 1 stand & &
\end{tabular}

Values: mean \pm 1 standard deviation (SD). CIMT Carotid Intima-Media Thickness. Advanced periodontitis is defined as periodontal pocket $\geq 6.0 \mathrm{~mm}$ 
Table 3 Odds ratios (ORs) and 95\% confidence intervals (Cls) for advanced periodontitis in relation to human $T$ cell leukemia virus-1 (HTLV-1) infections

\begin{tabular}{llll}
\hline & \multicolumn{2}{l}{ HTLV-1 infection } & $p$ \\
\cline { 2 - 3 } & $(-)$ & $(+)$ & \\
\hline No. of subjects & 744 & 163 & \\
No. of case (\%) & $160(21.5)$ & $37(22.7)$ & \\
Model 1 & 1.00 & $1.07(0.71,1.61)$ & 0.764 \\
Model 2 & 1.00 & $1.05(0.69,1.60)$ & 0.814 \\
Model 3 & 1.00 & $1.06(0.70,1.60)$ & 0.800 \\
\hline
\end{tabular}

Advanced periodontitis is defined as periodontal pocket $\geq 6.0 \mathrm{~mm}$. Atherosclerosis is defined as carotid intima-media thickness (CIMT) $\geq 1.1 \mathrm{~mm}$. Model 1: adjusted only for sex and age. Model 2: adjusted further for smoking status (never, former, current), number of remaining teeth, status of decayed teeth (presence, absence), body mass index (BMI), HDL-cholesterol, triglycerides, hemoglobin A1c (HbA1c), reticulocyte count, and CIMT. Model 3: adjusted further for antihyerlipidemic medication use (yes, no) and glucoselowering medication use (yes, no)

value could not reach a significant level, and essentially the same associations were observed for men and women.

\section{Association between reticulocytes and hypertension, and reticulocytes and atherosclerosis}

By using a sex- and age-adjusted model, analysis between tertiles of reticulocytes and hypertension, and between tertiles of reticulocytes and atherosclerosis was performed. Even the power did not reach significant value; reticulocyte count is positive tendency with hypertension and inverse tendency with atherosclerosis. Using the lowest tertile of reticulocyte count $\left(<4.390 \times 10^{4}\right.$ cells/ $\mu \mathrm{L}$ for men and $<4.140 \times 10^{4}$ cells $/ \mu \mathrm{L}$ for women) as a reference group, the adjusted ORs for hypertension and atherosclerosis were $1.08(0.77,1.50)$ and $0.93(0.66,1.32)$ for the second tertiles of reticulocyte count (4.390-5.724 $\times$ $10^{4} \mathrm{cells} / \mu \mathrm{L}$ for men and $4.140-5.551 \times 10^{4} \mathrm{cells} / \mu \mathrm{L}$ for women) and $1.21(0.86,1.70)$ and $0.89(0.62,1.27)$ for the highest tertile of reticulocyte count $\left(5.725 \times 10^{4}\right.$ cells $/ \mu \mathrm{L} \leq$ for men and $5.552 \times 10^{4}$ cells $/ \mu \mathrm{L} \leq$ for women) (not shown in table).

Sensitivity analysis shows that excluding participants with less than 10 remaining teeth, as reported in previous studies [6,9], yields trends similar to our major observations.

\section{Discussion}

In this study, we found no significant association between HTLV-1 infection and advanced periodontitis in elderly people without atherosclerosis. However, in elderly people with atherosclerosis, HTLV-1 infection was associated with an increased likelihood of advanced periodontitis.

Previously, we reported that HTLV-1 infection was positively related to advanced periodontitis among elderly participants with lower hematopoietic activity (lower reticulocyte count) but not among elderly participants with higher hematopoietic activity (higher reticulocyte count) [6]. Lower hematopoietic activity is the main cause of anemia in the elderly, and unexpected anemia in the elderly is characterized by the incidence of lowgrade inflammation [11]. In particular, elderly subjects suffering from anemia because of lower hematopoietic activity might be more susceptible to low-grade inflammation. Since erythrocytes possess antioxidant capacity [12] and activation of erythropoiesis can cause reduction in oxidative stress [13], anemia increases oxidative stress. Low-grade inflammation, shown to be well connected with oxidative stress [14], is also associated with periodontitis [32]. Therefore, the observed positive association between HTLV-1 infection and advanced periodontitis among elderly subjects with low reticulocyte counts could be due to the presence of low-grade inflammation.

Although in the present study the positive association between HTLV-1 infection and advanced periodontitis was observed only among participants with

Table 4 Odds ratios (ORs) and 95\% confidence intervals (Cls) for advanced periodontitis in relation to human T cell leukemia virus type-1 (HTLV-1) infections by status of atherosclerosis

\begin{tabular}{|c|c|c|c|c|c|c|c|}
\hline & \multicolumn{6}{|c|}{ Atherosclerosis } & \multirow{4}{*}{$\begin{array}{l}\text { Interaction } \\
p\end{array}$} \\
\hline & \multicolumn{3}{|l|}{$(-)$} & \multicolumn{3}{|l|}{$(+)$} & \\
\hline & \multicolumn{2}{|c|}{ HTLV-1 infection } & \multirow[t]{2}{*}{$p$} & \multicolumn{2}{|c|}{ HTLV-1 infection } & \multirow[t]{2}{*}{$p$} & \\
\hline & $(-)$ & $(+)$ & & $(-)$ & $(+)$ & & \\
\hline No. of subjects & 512 & 100 & & 232 & 63 & & \\
\hline No. of case (\%) & $108(21.1)$ & $15(15.0)$ & & $52(22.4)$ & $22(34.9)$ & & \\
\hline Model 1 & 1.00 & $0.64(0.35,1.15)$ & 0.135 & 1.00 & $2.02(1.09,3.73)$ & 0.026 & 0.015 \\
\hline Model 2 & 1.00 & $0.62(0.34,1.13)$ & 0.116 & 1.00 & $2.00(1.05,3.78)$ & 0.034 & 0.011 \\
\hline Model 3 & 1.00 & $0.61(0.34,1.12)$ & 0.113 & 1.00 & $2.01(1.06,3.81)$ & 0.033 & 0.013 \\
\hline
\end{tabular}

Advanced periodontitis is defined as periodontal pocket $\geq 6.0 \mathrm{~mm}$. Atherosclerosis is defined as carotid intima-media thickness (CIMT) $\geq 1.1 \mathrm{~mm}$. Model 1 : adjusted only for sex and age. Model 2: adjusted further for smoking status (never, former, current), number remaining teeth, status of decayed teeth (presence, absence), body mass index (BMI), HDL-cholesterol, triglycerides, hemoglobin A1c (HbA1c), reticulocyte count, and CIMT. Model 3: adjusted further for antihyerlipidemic medication use (yes, no) and glucose-lowering medication use (yes, no) 
Table 5 Sex-specific odds ratios (OR) and 95\% confidence intervals (Cls) for advanced periodontitis in relation to human T cell leukemia virus-1 (HTLV-1) infections by status of atherosclerosis

\begin{tabular}{|c|c|c|c|c|c|c|c|}
\hline & \multicolumn{6}{|c|}{ Atherosclerosis } & \multirow{4}{*}{$\begin{array}{l}\text { Interaction } \\
p\end{array}$} \\
\hline & \multicolumn{3}{|l|}{$\overline{(-)}$} & \multicolumn{3}{|l|}{$(+)$} & \\
\hline & \multicolumn{2}{|c|}{ HTLV-1 infection } & \multirow[t]{2}{*}{$p$} & \multicolumn{2}{|c|}{ HTLV-1 infection } & \multirow[t]{2}{*}{$p$} & \\
\hline & $\overline{(-)}$ & $(+)$ & & $\overline{(-)}$ & $(+)$ & & \\
\hline \multicolumn{8}{|l|}{ Men } \\
\hline No. of subjects & 186 & 31 & & 111 & 25 & & \\
\hline No. of case (\%) & $45(24.2)$ & $7(22.6)$ & & $29(26.1)$ & $12(48.0)$ & & \\
\hline Model 1 & 1.00 & $0.88(0.35,2.18)$ & 0.774 & 1.00 & $2.68(1.09,6.96)$ & 0.032 & 0.106 \\
\hline Model 2 & 1.00 & $0.79(0.30,2.05)$ & 0.624 & 1.00 & $3.39(1.24,9.24)$ & 0.017 & 0.071 \\
\hline Model 3 & 1.00 & $0.81(0.31,2.11)$ & 0.662 & 1.00 & $3.76(1.34,10.56)$ & 0.012 & 0.058 \\
\hline \multicolumn{8}{|l|}{ Women } \\
\hline No of subjects & 326 & 69 & & 121 & 38 & & \\
\hline No of case (\%) & $63(19.3)$ & $8(11.6)$ & & $23(19.0)$ & $10(26.3)$ & & \\
\hline Model 1 & 1.00 & $0.51(0.23,1.14)$ & 0.100 & 1.00 & $1.56(0.66,3.70)$ & 0.309 & 0.083 \\
\hline Model 2 & 1.00 & $0.51(0.23,1.16)$ & 0.108 & 1.00 & $1.46(0.57,3.69)$ & 0.430 & 0.111 \\
\hline Model 3 & 1.00 & $0.50(0.22,1.15)$ & 0.102 & 1.00 & $1.48(0.58,3.76)$ & 0.413 & 0.090 \\
\hline
\end{tabular}

Advanced periodontitis is defined as periodontal pocket $\geq 6.0 \mathrm{~mm}$. Atherosclerosis is defined as carotid intima-media thickness (CIMT) $\geq 1.1 \mathrm{~mm}$. Model 1: adjusted only for age. Model 2: adjusted further for smoking status (never, former, current), number of remaining teeth, status of decayed teeth (presence, absence), body mass index (BMI), HDL cholesterol, triglycerides, hemoglobin A1c (HbA1c), reticulocyte count, and CIMT. Model 3: adjusted further for antihyperlipidemic medication use (yes, no) and glucose-lowering medication use (yes, no)

atherosclerosis, the biological characteristics of HTLV-1 might explain the finding. HTLV-1 possesses a characteristic of inflammation inducer since the trans-activator protein of Tax of HTLV-1 enhances inflammation [7, 8]. The influence of HTLV-1 infection should be stronger in participants with inflammation than in those without inflammation. Since low-grade chronic inflammation causes progression of atherosclerosis [15] and disruption of microcirculation [16], the influence of HTLV-1 on endothelial (microvascular) dysfunction should be stronger for participants with atherosclerosis than that for participants without atherosclerosis. Further, severe cases of chronic periodontitis were recently reported to be associated with endothelial (microvascular) dysfunction $[9,10]$. Therefore, low-grade inflammation, but not hematopoietic activity itself and/or atherosclerosis, might be the culprit behind the reported data. This could explain the invariance of our major observations, even after necessary adjustments for reticulocyte and CIMT were incorporated in the data analysis.

However, as shown in Table 1, among participants with atherosclerosis, we found no significant differences in CIMT values between the HTLV-1 (-) and HTLV (+) groups. Among the participants with atherosclerosis, HTLV-1 (+) could have lower classical cardiovascular risk factors such as low HDL-cholesterol, high triglycerides, and high HbA1c than HTLV (-) which might also act as confounding factors. This suggests that despite clinical characteristics of HTLV-1 (+) subjects showing a lower risk of atherosclerosis progression than that of
HTLV-1 (-) subjects, the former might develop atherosclerosis. Further investigations with information on gingival microcirculation are necessary.

Furthermore, even the statistical power in this study could not reach a significant value among participants without atherosclerosis, compared to participants without HTLV-1 infection. Also, participants with HTLV-1 infection showed lower ORs in advanced periodontitis (Table 4) with higher CIMT values and higher reticulocyte values (Table 1 ).

Low-grade inflammation also induces hypertension [33]. Previously, we reported that height is an indicator of the capacity of vascular repair in elderly men [34], especially hypertensive men. In addition to that, elderly men with a short stature might have lower hematopoietic capacity evaluated by reticulocyte but have higher vascular remodeling activity than those with a high stature $[35,36]$. These studies have indicated that erythropoietic activity, estimated according to reticulocyte levels, could be an indicator of endothelial maintenance capacity and activity among elderly individuals.

A previous study with 2098 elderly participants reported that reticulocyte count was positively associated with hypertension, whereas it was inversely associated with atherosclerosis [31]. In our additional analyses, even the power could not reach significant value because of the small number of participants $(n=907)$, and reticulocyte count showed essentially the same tendency for hypertension and atherosclerosis. Hypertension is well known as a significant factor in endothelium injury, and 
atherosclerosis is a result of aggressive endothelial repair. Therefore, even a higher hematopoietic activity should have a higher tissue-repairing activity such as reduction of a marker of oxidative stress [13] and higher hematopoietic activity is also induced by more severe tissue injury (increased oxidative stress) [37, 38]. These associations indicate that the low level of reticulocytes itself indicates that low-grade inflammation tissue injury, such as advanced periodontitis, might elevate reticulocyte count. Therefore, no significant differences in reticulocyte counts between participants with and without advanced periodontitis were observed in this study (Table 2). Even though aging reduces this type of response activity resulting in tissue injury, it could not induce sufficient reticulocyte production. Therefore, anemia in the elderly is associated with low-grade inflammation [11]. Further investigation with larger samples and wider age ranges is necessary to clarify those associations.

Aging is a process that increases vascular aging-related inflammation [39]. Age-related decline of bone marrow activity (the productivity of hematopoietic stem cells) is associated with endothelial repair deficiency which results in lower CIMT $[40,41]$ and reticulocyte values [42]. Therefore, higher reticulocyte and CIMT values among elderly participants without atherosclerosis could have a beneficial influence on endothelium maintenance to some extent by promoting appropriate endothelial repair activity. Then, the inflammation inducer characteristics of HTLV-1 $[7,8]$ could have a beneficial influence on endothelial maintenance to some extent among elderly participants without atherosclerosis by stimulating bone marrow activity (the productivity of hematopoietic stem cells). Further investigation with information on hematopoietic stem cells is necessary.

Therefore, HTLV-1 infection that stimulates inflammation activity $[7,8]$ could have a beneficial association with advanced periodontitis among participants without atherosclerosis, but an unfavorable association with advanced periodontitis among participants with atherosclerosis. This might result in no significant association between HTLV-1 infection and advanced periodontitis among elderly participants (Table 3 ).

The clinical implication for the present study is that, even in non-symptomatic status, HTLV-1 infection could be associated with advanced periodontitis and atherosclerosis status might influence the association between HTLV-1 infection and advanced periodontitis among elderly people.

Potential limitations of this study warrant consideration. Although the age-related endothelial dysfunction may influence the association between HTLV-1 infection and advanced periodontitis, no data on the evaluation of endothelial function was available. Thus, further analyses that include endothelial function-related data such as flow-mediated dilation (FMD) will be necessary. This study did not include confirmatory tests for detection of HTLV-1. In spite of that, we believe that the influence of HTLV-1 was limited, especially since our previous study, using real-time reverse transcription-polymerase chain reaction with the hydrolysis probe and western blotting assays, showed only a small false positive rate $(1.2 \%)$ [43]. In addition, we have no information on the hygiene status of these samples. Previous studies have reported that the number of remaining teeth among the elderly Japanese depends on the type of dental visit [44]. Furthermore, tooth loss leads to reduced nutrient intake [45]. Therefore, we have used data on the number of remaining teeth as a confounding factor in our analysis. Further, we do not have the setup ready for evaluating potentially crucial cases of low-grade inflammation. The statistical value of sex-specific analyses is limited in the present study because of the small number of participants. However, essentially, the same associations were observed for men and women. Additionally, because this was a cross-sectional study, causal relationships cannot be established.

\section{Conclusion}

In conclusion, we found HTLV-1 infection to be positively associated with advanced periodontitis among elderly participants with atherosclerosis but not among those without atherosclerosis. Therefore, atherosclerosis might be a crucial factor that determines the association of HTLV-1 infection with advanced periodontitis among elderly Japanese people. These results were an efficient tool to clarify the role of the possible mechanism for the association between HTLV-1 and microcirculation.

\section{Abbreviations}

ANOVA: Analysis of variance; BMI: Body mass index; CIMT: Carotid intimamedia thickness; Cls: Confidence intervals; CLEIA: Chemiluminescent enzyme immunoassay; FMD: Flow-mediated dilation; HDL: High-density lipoprotein; HTLT-1: Human T cell leukemia virus type-1; ORs: Odds ratios; RBC: Red blood cell

\section{Acknowledgements}

We are grateful to the staff of Goto City office for their outstanding support. We would like to thank Editage (www.editage.jp) for English language editing.

\section{Authors' contributions}

YS designed the study, performed the statistical analyses, interpreted the data, and drafted and revised the manuscript. HY, MK, RF, TI, HF, HH, KoK, KaK, SYK, and TS assisted with the study design, were involved in data collection, and checked the manuscript. HY, SYK, AK, and TM participated in the study concept and checked the manuscript. TM was the general coordinator and designed the study. All authors read and approved the final manuscript.

\section{Funding}

This study was supported by Grants-in-Aid for Scientific Research from the Japan Society for the Promotion of Science (no. 18K06448, no. 17H03740). 


\section{Availability of data and materials}

The datasets generated during and/or analyzed during the current study are not publicly available due to ethical consideration but are available from the corresponding author on reasonable request.

\section{Ethics approval and consent to participate}

This study was approved by the Ethics Committee of Nagasaki University Graduate School of Biomedical Sciences (project registration number 14051404). All procedures involving human participants were performed in accordance with the ethical standards of the institution research committee and with the 1964 Helsinki Declaration and its later amendments for comparable ethical standards.

\section{Consent for publication}

Not applicable

\section{Competing interests}

The authors declare that they have no competing interests.

\section{Author details}

'Department of Community Medicine, Nagasaki University Graduate School of Biomedical Sciences, Nagasaki-shi, Sakamoto 1-12-4, Nagasaki 852-8523, Japan. ${ }^{2}$ Department of Cardiovascular Disease Prevention, Osaka Center for Cancer and Cardiovascular Disease Prevention, Osaka, Japan. ${ }^{3}$ Department of General Medicine, Nagasaki University Hospital, Nagasaki, Japan. ${ }^{4}$ Department of Oral Health, Nagasaki University Graduate School of Biomedical Sciences, Nagasaki, Japan. ${ }^{5}$ Department of Community Oral Health, School of Dentistry, Asahi University, Gifu, Japan. ${ }^{6}$ Community Medical Network Center, Nagasaki University Hospital, Nagasaki, Japan. ${ }^{7}$ Department of Immunology and Rheumatology, Nagasaki University Graduate School of Biomedical Sciences, Nagasaki, Japan. ${ }^{8}$ Department of Island and Community Medicine, Nagasaki University Graduate School of Biomedical Sciences, Nagasaki, Japan.

Received: 7 October 2019 Accepted: 5 December 2019

Published online: 28 December 2019

\section{References}

1. Gascoyne RD. HTLV-1: a significant retrovirous. Can Fam Physician. 1988;34: 2513-7.

2. Shoeibi A, Rafatpanah H, Azarpazhooh A, Mokhber N, Hedayati-Moghaddam MR, Amiri A, et al. Clinical features of HTLV-1-associated myelopathy/tropical spastic paraparesis (HAM/TSP) in northeast Iran. Acta Neurol Belg. 2013; 113(4):427-33

3. Yamaguchi K, Watanabe T. Human T lymphotropic virus type-l and adult Tcell leukemia in Japan. Int J Hematol. 2002;76(Suppl 2):240-5.

4. Murphy EL, Hanchard B, Figueroa JP, Gibbs WN, Lofters WS, Campbell $M$, et al. Modelling the risk of adult T-cell leukemia/lymphoma in persons infected with human T-lymphotropic virus type I. Int J Cancer. 1989:43(2):250-3.

5. Kaplan JE, Osame M, Kubota H, Igata A, Nishitani H, Maeda Y, et al. The risk of development of HTLV-I-associated myelopathy/tropical spastic paraparesis among persons infected with HTLV-I. J Acquir Immune Defic Syndr. 1990;3(11):1096-101.

6. Shimizu Y, Yamanashi H, Kitamura M, Furugen R, Iwasaki T, Fukuda $H$, et al. Association between human T cell leukemia virus 1 (HTLV-1) infection and advanced periodontitis in relation to hematopoietic activity among elderly participants: a cross-sectional study. Environ Health Prev Med. 2019;24(1):42.

7. Fochi S, Mutascio S, Bertazzoni U, Zipeto D, Romanelli MG. HTLV deregulation of the NF-KB pathway: an update on Tax and antisense proteins role. Front Microbiol. 2018;9:285.

8. Hanon E, Asquith RE, Taylor GP, Tanaka Y, Weber JN, Bangham CR. High frequency of viral protein expression in human $T$ cell lymphotropic virus type 1-infected peripheral blood mononuclear cells. AIDS Res Hum Retroviruses. 2000;16(16):1711-5.

9. Hayashida H, Saito T, Kawasaki K, Kitamura M, Furugen R, Iwasaki T, et al. Association of periodontitis with carotid artery intima-media thickness and arterial stiffness in community-dwelling people in Japan: the Nagasaki Islands study. Atherosclerosis. 2013;229(1):186-91.

10. Lira-Junior R, Figueredo CM, Bouskela E, Fischer RG. Severe chronic periodontitis is associated with endothelial and microvascular dysfunctions: a pilot study. J Periodontol. 2014;85(12):1648-57.
11. Artz AS, Xue QL, Wickrema A, Hesdorffer C, Ferrucci L, Langdon JM, et al. Unexplained anaemia in the elderly is characterised by features of low grade inflammation. Br J Haematol. 2014;167(2):286-9.

12. Grune T, Sommerburg O, Siems WG. Oxidative stress in anemia. Clin Nephrol. 2000;53(1 Suppl):S18-22.

13. Noguchi-Sasaki M, Sasaki Y, Matsuo-Tezuka Y, Yasuno H, Kurasawa M, Yorozu K, et al. Reduction of a marker of oxidative stress with enhancement of iron utilization by erythropoiesis activation following epoetin beta pegol administration in iron-loaded db/db mice. Int J Hematol. 2016;103(3):26273.

14. Wu J, Xia S, Kalionis B, Wan W, Sun T. The role of oxidative stress and inflammation in cardiovascular aging. Biomed Res Int. 2014;2014:615312.

15. Geng S, Chen K, Yuan R, Peng L, Maitra U, Diao N, et al. The persistence of low-grade inflammatory monocytes contributes to aggravated atherosclerosis. Nat Commun. 2016;7:13436.

16. Payne GW. Effect of inflammation on the aging microcirculation: impact on skeletal muscle blood flow control. Microcirculation. 2006;13(4):343-52.

17. Ong G. Periodontal reasons for tooth loss in an Asian population. J Clin Periodontol. 1996;23(4):307-9.

18. Gessain A, Cassar O. Epidemiological aspects and world distribution of HTLV-1 infection. Front Microbiol. 2012;3:388.

19. Morita M, Kimura T, Kanegae M, Ishikawa A, Watanabe T. Reasons for extraction of permanent teeth in Japan. Community Dent Oral Epidemiol. 1994;22(5):303-6.

20. Hara T, Takamura N, Akashi S, Nakazato M, Maeda T, Wada M, et al. Evaluation of clinical markers of atherosclerosis in young and elderly Japanese adults. Clin Chem Lab Med. 2006;44(7):824-9.

21. Kawamori R, Yamasaki Y, Matsushima H, Nishizawa H, Nao K, Hougaku H, et al. Prevalence of carotid atherosclerosis in diabetic patients. Ultrasound high-resolution B-mode imaging on carotid arteries. Diabetes Care. 1992; 15(10):1290-4.

22. Brown LJ, Brunelle JA, Kingman A. Periodontal status in the United States, 1988-1991: prevalence, extent, and demographic variation. J Dent Res. 1996; 75:672-83.

23. Nociti FH Jr, Casati MZ, Duarte PM. Current perspective of the impact of smoking on the progression and treatment of periodontitis. Periodontol 2000. 2015;67(1):187-210.

24. Saotome $Y$, Tada A, Hanada N, Yoshihara A, Uematsu H, Miyazaki $H$, et al. Relationship of cariogenic bacteria levels with periodontal status and root surface caries in elderly Japanese. Gerodontology. 2006;23(4): 219-25.

25. Frentzen M, Schüler N, Nolden R. Correlation between caries prevalence (DMFS) and periodontal condition (CPITN) in more than 2000 patients. Int Dent J. 1990:40(5):313-8.

26. Deshpande NC, Amrutiya MR. Obesity and oral health - Is there a link? an observational study. J Indian Soc Periodontol. 2017;21(3):229-33.

27. Almeida Abdo J, Cirano FR, Casati MZ, Ribeiro FV, Giampaoli V, Casarin RCV, et al. Influence of dyslipidemia and diabetes mellitus on chronic periodontal disease. J Periodontol. 2013;84(10):1401-8.

28. Lee JB, Yi HY, Bae KH. The association between periodontitis and dyslipidemia based on the Fourth Korea National Health and Nutrition Examination Survey. J Clin Periodontol. 2013;40(5):437-42.

29. Preshaw PM, Alba AL, Herrera D, Jepsen S, Konstantinidis A, Makrilakis K, et al. Periodontitis and diabetes: a two-way relationship. Diabetologia. 2012; 55(1):21-31

30. Bradley AD, Zhang $Y$, Jia Z, Zhao G, Wang X, Pranke L, et al. Effect of simvastatin prodrug on experimental periodontitis. J Periodontol. 2016;87(5): $577-82$.

31. Shimizu Y, Kawashiri SY, Yamanashi H, Koyamatsu J, Fukui S, Kondo H, et al. Reticulocyte levels have an ambivalent association with hypertension and atherosclerosis in the elderly: a cross-sectional study. Clin Interv Aging. 2019; 14:849-57.

32. Josey MJ, Merchant AT. Low-grade systemic inflammation may increase the risk of periodontitis. J Evid Based Dent Pract. 2016;16(4):251-3.

33. Madej A, Dąbek J, Majewski M, Szuta J. Effect of perindopril and bisoprolo on IL-2, INF-y, hs-CRP and T-cell stimulation and correlations with blood pressure in mild and moderate hypertension. Int I Clin Pharmacol Ther. 2018:56(9):393-9.

34. Shimizu Y, Sato S, Koyamatsu J, Yamanashi H, Nagayoshi M, Kadota K, et al. Height is an indicator of vascular maintenance capacity in older men. Geriatr Gerontol Int. 2017;17(10):1729-36. 
35. Shimizu Y, Sato S, Koyamatsu J, Yamanashi H, Nagayoshi M, Kadota K, et al. Height indicates hematopoietic capacity in elderly Japanese men. Aging (Albany NY). 2016;8(10):2407-13.

36. Shimizu Y, Sato S, Koyamatsu J, Yamanashi H, Nagayoshi M, Kadota K, et al. Possible mechanism underlying the association between height and vascular remodeling in elderly Japanese men. Oncotarget. 2017;9(8):774957.

37. Gafter-Gvili A, Zingerman B, Rozen-Zvi B, Ori Y, Green H, Lubin I, et al. Oxidative stress-induced DNA damage and repair in human peripheral blood mononuclear cells: protective role of hemoglobin. PLoS One. 2013; 8(7):e68341.

38. Zhao JL, Baltimore D. Regulation of stress-induced hematopoiesis. Curr Opin Hematol. 2015;22(4):286-92.

39. Chiriacò M, Georgiopoulos G, Duranti E, Antonioli L, Puxeddu I, Nannipieri $M$, et al. Inflammation and vascular ageing: from telomeres to novel emerging mechanisms. High Blood Press Cardiovasc Prev. 2019;26(4):321-9.

40. Shimizu Y, Yamanashi H, Noguchi Y, Koyamatsu J, Nagayoshi M, Kiyoura K, et al. Cardio-ankle vascular index and circulating CD34-positive cell levels as indicators of endothelial repair activity in older Japanese men. Geriatr Gerontol Int. 2019;19(6):557-62.

41. Shimizu Y, Yamanashi H, Noguchi Y, Koyamatsu J, Nagayoshi M, Kiyoura K, et al. Association between chronic kidney disease and carotid intima-media thickness in relation to circulating CD34-positive cell count among community-dwelling elderly Japanese men. Atherosclerosis. 2019;283:85-91.

42. Guralnik JM, Ershler WB, Schrier SL, Picozzi VJ. Anemia in the elderly: a public health crisis in hematology. Hematology Am Soc Hematol Educ Program. 2005:528-32.

43. Yamanashi H, Koyamatsu J, Nagayoshi M, Shimizu Y, Kawashiri SY, Kondo H, et al. Human T-cell leukemia virus-1 infection is associated with atherosclerosis as measured by carotid intima-media thickness in Japanese community-dwelling older people. Clin Infect Dis. 2018;67(2):291-4.

44. Saito M, Shimazaki Y, Nonoyama T, Tadokoro Y. Type of dental visit and number of remaining teeth in Japanese elders. J Oral Sci. 2018;60(4):611-7.

45. Kosaka T, Kida M. Tooth loss leads to reduced nutrient intake in middle-aged and older Japanese individuals. Environ Health Prev Med. 2019;24(1):15.

\section{Publisher's Note}

Springer Nature remains neutral with regard to jurisdictional claims in published maps and institutional affiliations.

Ready to submit your research? Choose BMC and benefit from:

- fast, convenient online submission

- thorough peer review by experienced researchers in your field

- rapid publication on acceptance

- support for research data, including large and complex data types

- gold Open Access which fosters wider collaboration and increased citations

- maximum visibility for your research: over $100 \mathrm{M}$ website views per year

At $\mathrm{BMC}$, research is always in progress.

Learn more biomedcentral.com/submissions 